\title{
The Research and design of high-frequency differential signal isolation circuit in the differential probe
}

\author{
Chao Chen ${ }^{1, a}$, Feng Sun ${ }^{2, b}$, Lei Cao ${ }^{2, c}$, Yusai Zheng ${ }^{2}$ \\ ${ }^{1}$ Naval Equipment Research Institute, Beijing, 102200, China \\ ${ }^{2}$ School of Mechanical, Electrical \& Information Engineering, Shandong University, Weihai, 264209, \\ China \\ aemail: 13801114129@139.com, bemail: sunfeng890526 @163.com, email: caolei1993@126.com
}

Keywords: Differential probe; Differential signal; Isolation

\begin{abstract}
When we use oscilloscopes to observe power waveforms, inverter circuit waveforms and other high voltage signal waveforms through conventional passive probes, there are many risk factors because of grounding problem. However, we can use high voltage differential probe to solve these problems. The key technology of differential probe is to design high frequency differential isolation circuit [1]. This paper designs a high-speed differential signal transmission circuit with theoretical analysis, simulation and testing, enabling the transmission frequency up to $10 \mathrm{MHz}$.
\end{abstract}

\section{Introduction}

With the rapid development of computers, communications, and electronics industry, signal transmission rate continuously improves. At the same time many signal protocols have been improved from the single-ended topology to differential topology. As one of species in active high voltage, differential probe plays an irreplaceable role. The single-ended voltage probes, which is used to measure the voltage waveform of the circuit in a node, and the node voltage is relative to earth; however, differential probe measures the relative voltage of the two signals, which has nothing to do with the earth. Due to some signal characteristics, signals can be measured by differential probe without many security risks. The basic circuit of a differential probe is high-speed differential signal isolation circuit [2].

\section{The Differential Probe Model}

\section{Conventional model of the differential probe.}

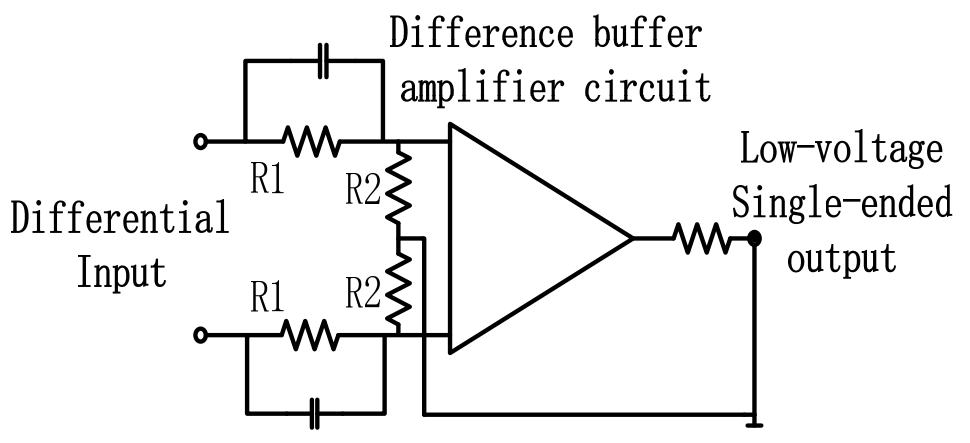

Fig.1 Conventional schematic of the differential probe

In Figure 1, R1 has a pretty high resistance, and it is usually set to megohm. The resistance of R2 is usually set to kohm, which will produce a great resistance between the high voltage input and earth. To a certain extent, this approach may avoid some dangers caused by the single-ended probe. However,we can see from Figure 1 that it was not completely isolated between the high voltage input and output, and only employed the high value of R1 to act as a safety barrier between the high input and low output. 


\section{Improved model of the differential probe.}

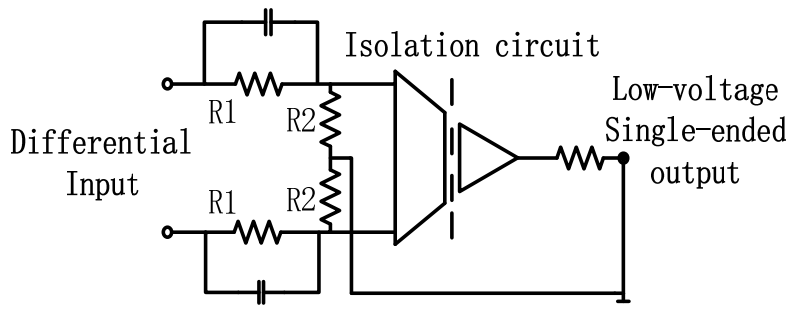

Fig.2 Improved model of differential probe with electrically isolated

In Figure 2, the isolation circuit has been added on the conventional differential probe. Between the high voltage input and low output, there is not only the high value of resistance, but also the isolation circuit. In this way, it can realize the real electrical isolation between the high voltage input and low output, and prevent damages on the oscilloscope. Finally it ensures the personal safety of the measurement personnel.

Design of High-speed Differential Signal Isolation Circuit [3]

Test of optocoupler isolation circuit.

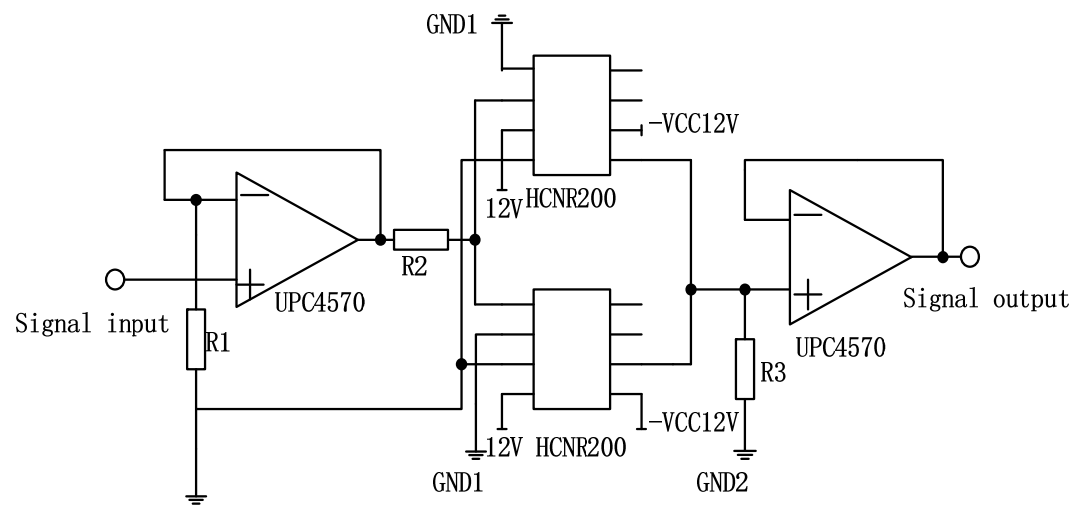

Fig.3 The schematic of isolation circuit

The circuit consists two pieces of optocouplers.

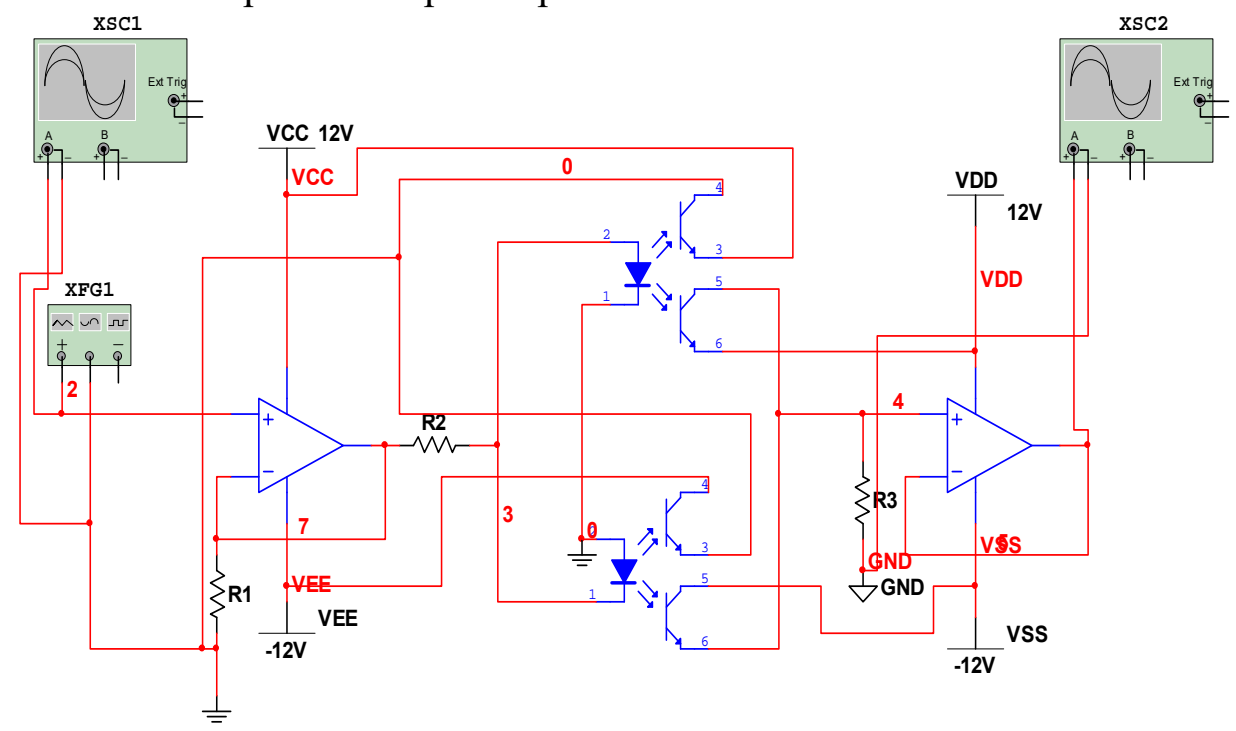

Fig. 4 The simulation circuit of optocoupler

The simulation results are shown in Figure 5 (a) and (b), Figure 5 (a) is the square wave test signal whose frequency is $2 \mathrm{MHz}$ and the peak-to-peak value is $4 \mathrm{~V}$. Figure 5 (b) is the output signal after optocoupler isolation circuit. 


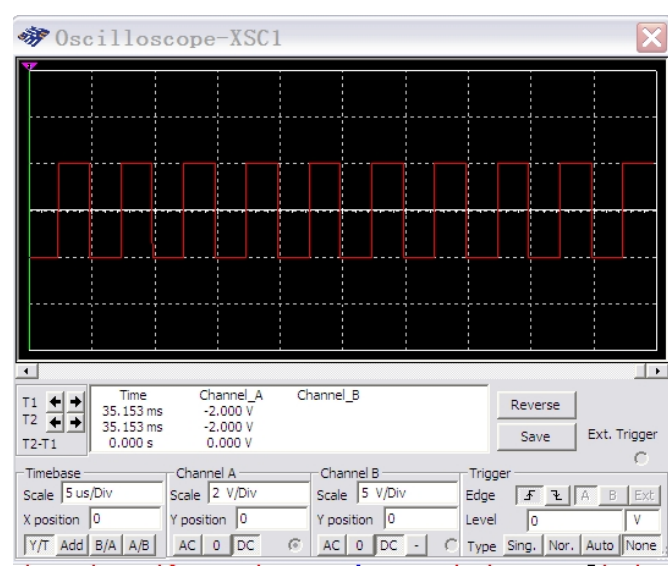

Fig. 5 (a)

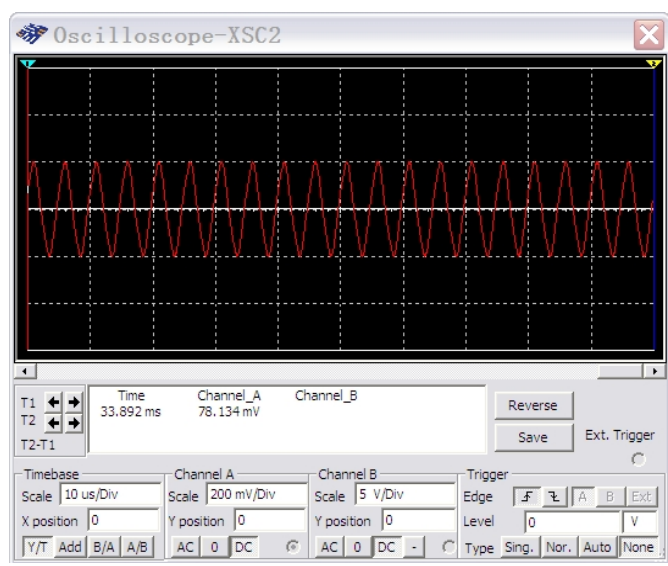

Fig. 5 (b)

The results show that the transmission effect of the optocoupler isolation circuit is not ideal with the distortion of the signal happening. Therefore, this paper argues that the optocoupler isolation circuit is not feasible.

Two operational amplifiers isolation circuit.

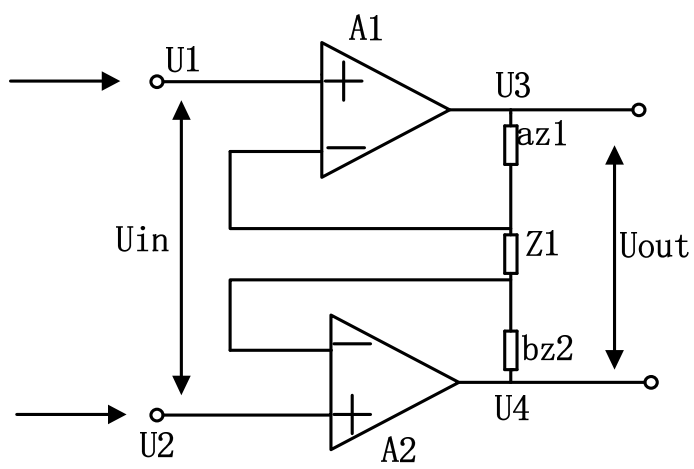

Fig. 6 Two operational amplifiers isolation circuit

The circuit is composed of two operational amplifiers, which has high input impedance and can transmit differential signal. We can calculate the transfer function between the input and output by the characteristics of the operational amplifier [4].

$$
\begin{aligned}
& \frac{U 1-U 2}{Z 1}=\frac{U 3-U 1}{a Z 1} \\
& \frac{U 1-U 2}{Z 1}=\frac{U 2-U 4}{b Z 2}
\end{aligned}
$$

The relationship between the input voltage and output voltage can be calculated through the Formula 1 and Formula 2.

$U 3-U 4=(1+a+b)(U 1-U 2)$

The Formula 3 shows that we can satisfy certain proportion relationship between the input voltage and output voltage by adjusting the resistance. 


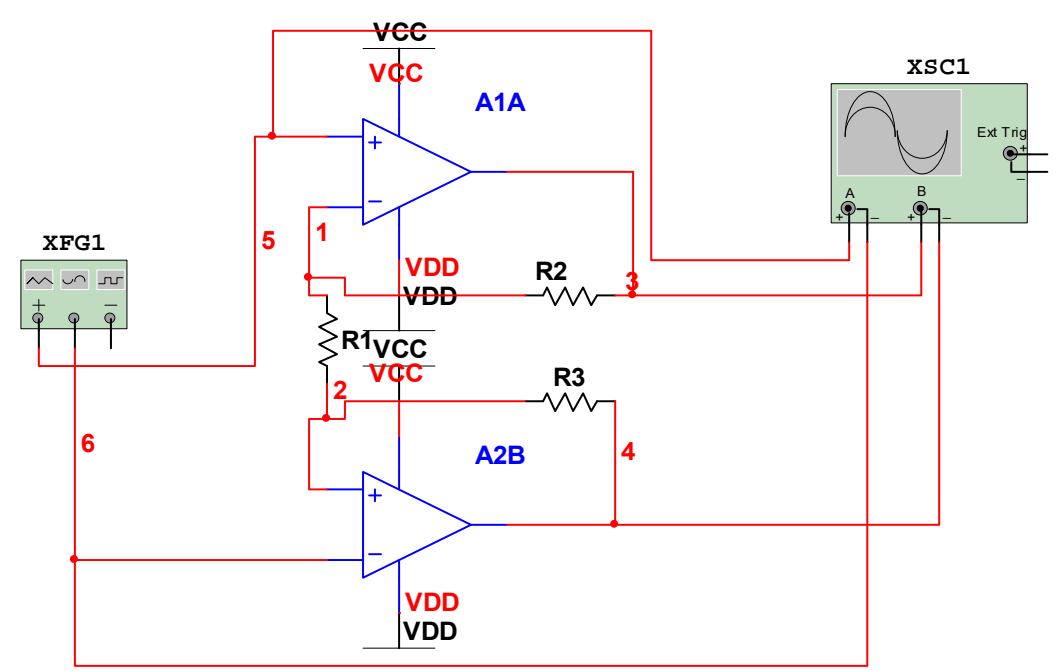

Fig.7 Dual operational amplifiers isolation circuit

Similarly, the transmission waveform distortion is serious, so this paper argues that this plan is not workable.

JFET differential amplifier isolation circuit [5].

Differential amplifier circuit principle:

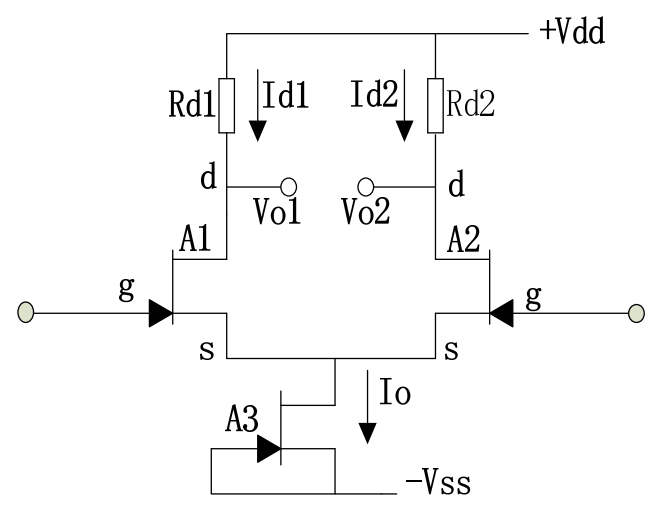

Fig. 8 JFET differential amplifier isolation circuit

When differential signals are put in, its voltage gain of input and output is:

$A_{v d}=\frac{V_{o 1}-V_{o 2}}{V_{i d}}=-g_{m} R_{d}$

$g_{m}$ is the transconductance between $\mathrm{A} 1$ and $\mathrm{A} 2, R_{d}$ is the resistance of $R_{d 1}$ and $R_{d 2}, V_{i d}$ is the differential signal input. This circuit has a high common mode rejection ratio.

$$
K_{C M R}=\left|\frac{A_{v d}}{A_{v c}}\right|
$$

The Formula (5) shows the smaller value of $A_{v C}$ (the common mode voltage gain) and the bigger value of $A_{V D}$ (the differential voltage gain), the value of $K_{C M R}$ (common mode rejection ratio) which represents the strong ability to suppress the common mode signal. When running in dual output mode, the value of the common mode voltage gain is close to zero and the common mode rejection ratio is very large. The differential amplifier circuit is composed of JEFT in which the input resistance is able to reach $10^{12} \Omega$. At the same time the JFET allows the transmission of high frequency signals. This paper designs a simulation circuit (as shown in Figure 9) to test the isolation effect of the differential amplifier circuit. As the differential output voltage signal of JFETS differential amplifier circuit is too small to cause measurement error, this paper adds two transistors based on JFET differential amplifier circuit in order to significantly increase the output differential 
voltage.

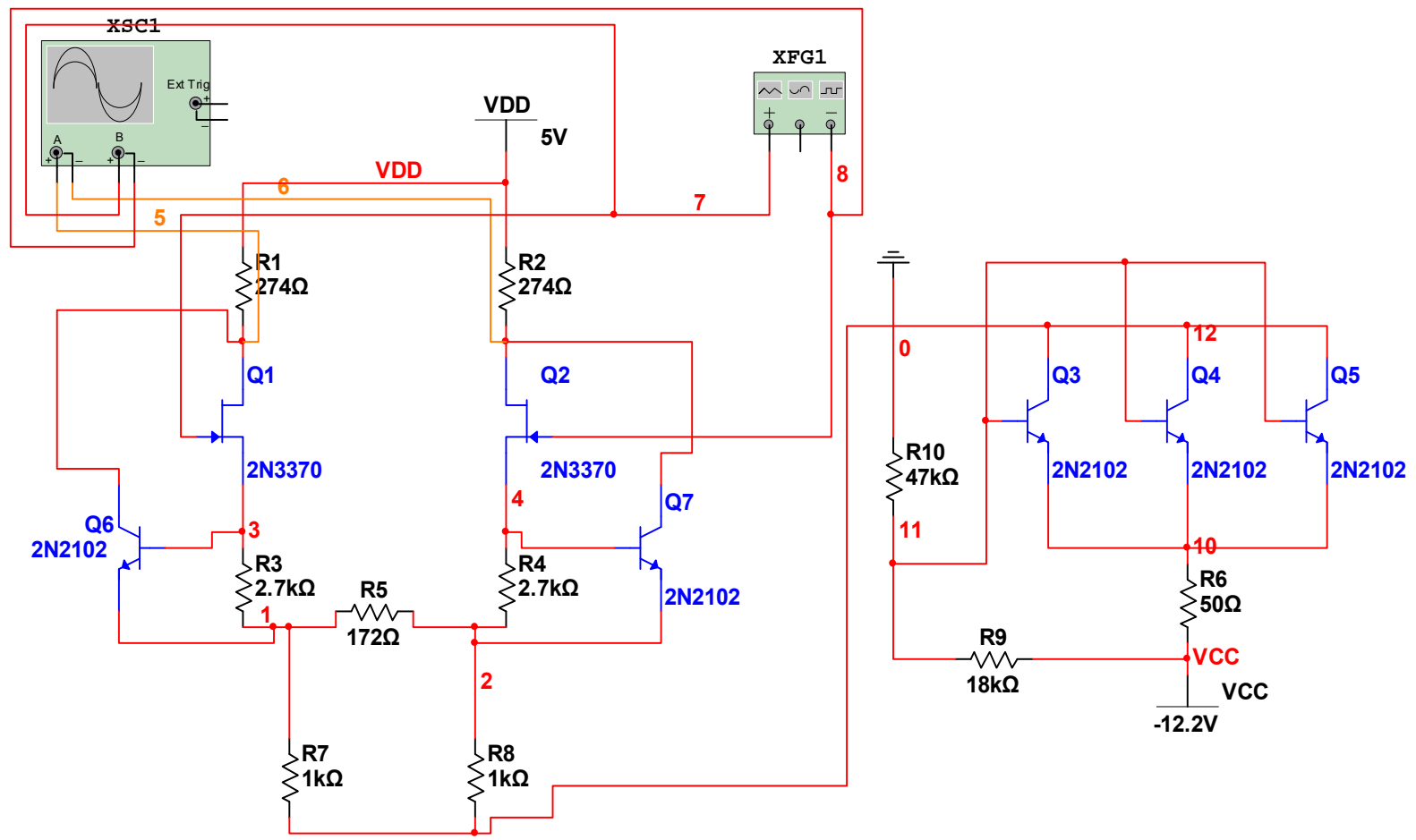

Fig.9 JFET differential amplifier isolation circuit

The collector current of the three transistors Q3、Q4、Q5 operating in the saturation region is essentially unchanged, and the three transistors form a simple constant current source circuit, which provides the bias voltage to the junction type field effect transistor. The ratio between differential output voltage and the differential input voltage can be adjusted by the resistance of R5, the ratio was adjusted to $1: 1$ in this paper.

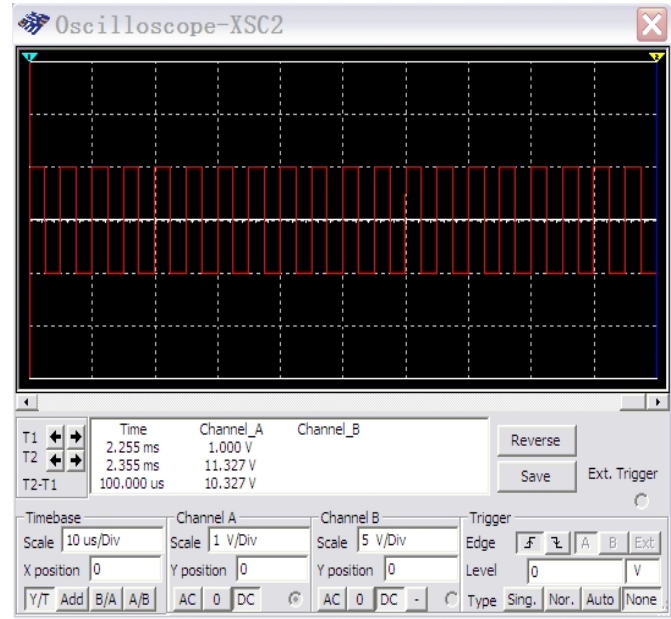

Fig.10 (a)

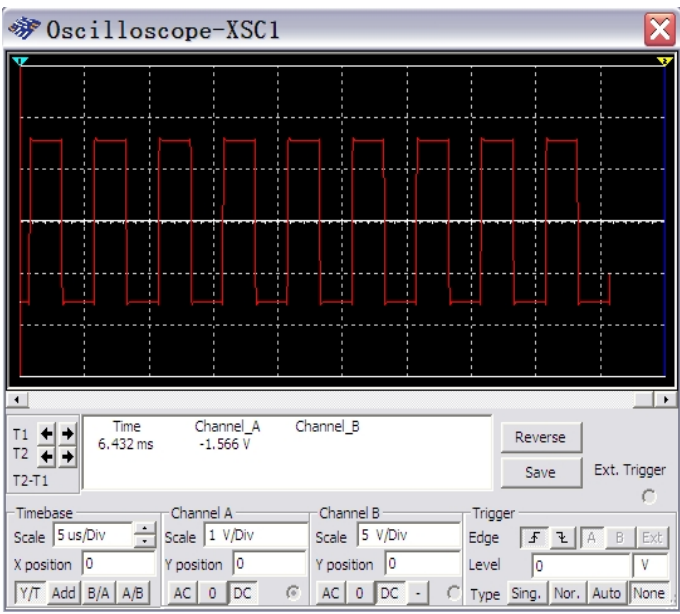

Fig.10 (b)

Figure10 (a) is the input signal, and Figure10 (b) is the output signal. The simulation results show that there is no waveform distortion, and the frequency of the $10 \mathrm{MHz}$ signal can be transmitted through the test circuit established in this paper. 


\section{The actual test}

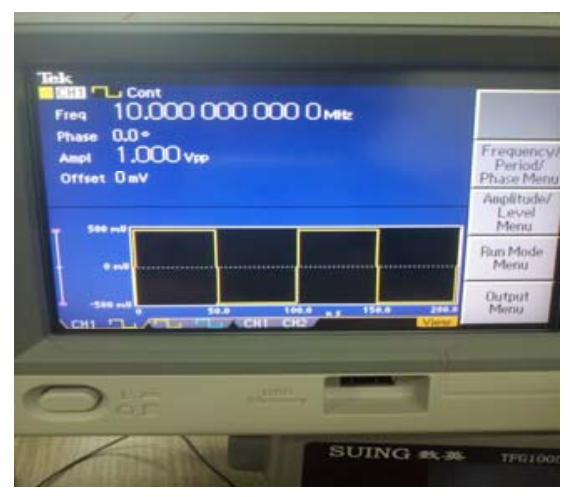

Fig.11 (a) The input signal

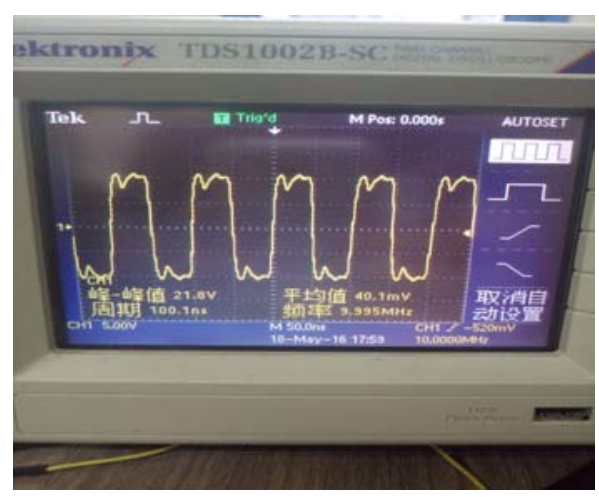

Fig.11 (b) The output signal

Figure11 (a) is the input signal produced by the power function generator which needs to connect with power resistor, and its frequency is $10 \mathrm{MHz}$. Figure 11 (b) is the output signal, and we can see just a little distortion in the waveform by the graphics.

\section{Conclusion}

This paper designs a high-speed differential signal transmission circuit with theoretical analysis, simulation and testing, enabling the transmission frequency up to $10 \mathrm{MHz}$. The active differential probe is an indispensable instrument in the measurement of differential signal. The high speed differential signal isolation circuit is a key part of the differential probe, which directly affects the performance of the differential probe.

\section{Acknowledgement}

On the occasion of the completion of this thesis, thank my colleagues for their help and thank my students for my work.

\section{References}

[1] Schamel,Al. Differential amplifier provides accurate, safe oscilloscope measurements. Powerconversion and Intelligent Motion, 1996.

[2] Anon. Sequential high voltage probe tester. IBM technical disclosure bulletin, 1986:4494-4495.

[3] Peng Jiang, a typical differential amplifier circuit design and test [J] ITS Applications, 2010,20: 25-28.

[4] Van Den Bossche, Alex. Two channel high voltage differential probe for power electronics applications. European Conference on Power Electronics and Applications, 2013.

[5] Phillips, Thomas A. Ferroelectric field-effect transistor differential amplifier circuit analysis. Integrated Ferroelectrics, 2009:107-117. 